\title{
Genotypes and public health potential of Enterocytozoon bieneusi and Giardia duodenalis in crab-eating macaques
}

$\mathrm{Li} \mathrm{Chen}^{1 \dagger}$, Jianguo Zhao ${ }^{2 \dagger}, \mathrm{Na} \mathrm{Li}^{3}$, Yaqiong Guo ${ }^{3}$, Yuanyuan Feng ${ }^{1}$, Yaoyu Feng ${ }^{1,3^{*}}$ and Lihua Xiao ${ }^{3^{*}}$

\begin{abstract}
Background: Enterocytozoon bieneusi and Giardia duodenalis are common human and animal pathogens. Studies have increasingly shown that non-human primates (NHPs) are common hosts of these two zoonotic parasites. However, few studies have explored the genetic diversity and public health potential of these pathogens in laboratory monkeys. In this study, we examined the genetic diversity of the two pathogens in crab-eating macaques (Macaca fascicularis) in a commercial facility in Hainan, China.
\end{abstract}

Results: Enterocytozoon bieneusi and G. duodenalis were detected by PCR analysis in 461/1452 (31.7\%) and 469/1452 (32.3\%) fecal specimens from the animals, respectively. Significantly higher detection rates of E. bieneusi were detected in males $(36.5 \%, 258 / 706)$ than in females $\left(26.7 \%, 160 / 599 ; X^{2}=14.391, P=0.0001\right)$, in animals with loose stools $(41.4 \%, 151 / 365)$ than those with normal stool $\left(28.5 \%, 310 / 1087 ; X^{2}=20.83, P<0.0001\right)$, and in animals of over 3 years of age $(38.6 \%, 135 / 350)$ than those of $1-3$ years $\left(29.6 \%, 326 / 1,102 ; X^{2}=9.90, P=0.0016\right)$. For $G$. duodenalis, the detection rate in males $(33.4 \%, 236 / 706)$ was higher than in females but not statistically significant $(30.2 \%, 181 / 599$; $\left.X^{2}=1.54, P=0.2152\right)$, in monkeys with loose stools $(41.1 \%, 150 / 365)$ than those with normal stools $(29.3 \%, 319 / 1087$; $\left.X^{2}=17.25, P<0.0001\right)$, and in monkeys of $1-3$ years of age $(36.6 \%, 403 / 1102)$ than those over 3 years $(18.9 \%, 66 / 350$; $\left.X^{2}=38.11, P<0.0001\right)$. Nine E. bieneusi genotypes were detected in this study by DNA sequence analysis of the internal transcribed spacer of the rRNA gene, namely Type IV (236/461), Peru8 (42/461), Pongo2 (27/461), Peru11 (12/461), D (4/461) and PigEblTS7 (1/461) previously seen in NHPs as well as humans, and CM1 (119/461), CM2 (17/461) and CM3 (3/461) that had been only detected in NHPs. DNA sequence analyses of the tpi, gdh and bg loci identified all G. duodenalis specimens as having assemblage B. Altogether, eight ( 4 known and 4 new), seven ( 6 known and 1 new) and seven (4 known and 3 new) subtypes were seen at the tpi, gdh and bg loci, leading to the detection of 53 multi-locus genotypes (MLG-B-hn01 to MLG-B-hn53). Most of them were genetically related to those previously seen in common Old-World monkeys.

Conclusions: Data from this study indicate a common occurrence of zoonotic genotypes of E. bieneusi and assemblage B of $G$. duodenalis in farmed crab-eating macaques in Hainan, China.

Keywords: Enterocytozoon bieneusi, Giardia duodenalis, Genotypes, Multi-locus genotyping, Crab-eating macaques

\footnotetext{
*Correspondence: yyfeng@ecust.edu.cn; Ixiao1961@gmail.com

${ }^{\dagger} \mathrm{Li}$ Chen and Jianguo Zhao contributed equally to this work

'State Key Laboratory of Bioreactor Engineering, School of Resource and Environmental, East China University of Science and Technology,

Shanghai 200237, China

${ }^{3}$ College of Veterinary Medicine, South China Agricultural University,

Guangzhou 510642, China

Full list of author information is available at the end of the article
}

C The Author(s) 2019. This article is distributed under the terms of the Creative Commons Attribution 4.0 International License (http://creativecommons.org/licenses/by/4.0/), which permits unrestricted use, distribution, and reproduction in any medium, provided you give appropriate credit to the original author(s) and the source, provide a link to the Creative Commons license, and indicate if changes were made. The Creative Commons Public Domain Dedication waiver (http://creativecommons.org/ publicdomain/zero/1.0/) applies to the data made available in this article, unless otherwise stated. 


\section{Background}

Giardia duodenalis and Enterocytozoon bieneusi are common human pathogens. At present, there are more than 200 million of annual giardiasis cases in humans, while microsporidiosis is a common cause of diarrhea $[1,2]$. The incidence of giardiasis has been reported to be 5.5 per 100,000 people in the UK and 7.5 per 100,000 people in the USA [3]. In China, almost 30 million people are infected with G. duodenalis every year [2]. For $E$. bieneusi, the detection rates range between $2-78 \%$ in industrialized and developing countries [4-6]. Over 200 giardiasis outbreaks have been reported in the world during the period 2004-2016, while E. bieneusi also caused an outbreak in France [6-9].

Non-human primates (NHPs) are important experimental animals in public health research because of their high genetic similarity to humans [10]. A growing number of studies have found that NHPs are the hosts of many parasites, including gastrointestinal protists $E$. bieneusi and G. duodenalis, which are transmitted in similar fecal-oral routes $[2,11]$. In addition to affecting the health and wellbeing of these laboratory animals, both pathogens are potentially zoonotic, causing diseases in humans $[12,13]$.

Of the 17 known human-pathogenic microsporidian species, E. bieneusi is the most common [14]. Based on sequence analysis of the internal transcribed spacer (ITS) of the rRNA gene, more than 200 E. bieneusi genotypes have been identified [15]. In phylogenetic analysis, these E. bieneusi genotypes are divided into at least 11 groups [16]. Among them, Group 1 contains most genotypes found in humans and many genotypes in animals, thus is considered to be the zoonotic group. In contrast, Groups 2-11 include genotypes found in specific groups of hosts, including humans, thus are considered more host-specific. There is also a so-called outlier group in dogs [1722]. Thus, genotyping E. bieneusi in NHPs can help us understand the zoonotic potential of E. bieneusi in these animals.

At present, more than $50 \mathrm{E}$. bieneusi genotypes have been found in NHPs, most of which belong to Group 1 [23]. Among them, genotypes A, D, Type IV, EbpC, Peru7, Peru8, Peru11, PigEBITS7, Henan-V, WL15, I and BEB6 have been found in humans in several countries, including China $[6,13,15,18,24-30]$. Of these, genotypes $\mathrm{A}$ and I appear to be more common in diarrheic children in China than other genotypes, suggesting that there could be differences in infectivity or virulence among E. bieneusi genotypes [6,31]. Therefore, NHPs are potential reservoir hosts for zoonotic transmission of $E$. bieneusi.

Similarly, eight distinct G. duodenalis assemblages (A-H) have been identified by genetic analysis of triosephosphate isomerase $(t p i)$, ssrRNA, $\beta$-giardin $(b g)$, glutamate dehydrogenase $(g d h)$ and other genes [2, 32, 33]. Among them, assemblages A and B are most commonly found in humans and NHPs, assemblages $C$ and $\mathrm{D}$ are mainly detected in canines, assemblage $\mathrm{E}$ mainly infects ruminants and other hoofed animals, whereas assemblages F, G and $\mathrm{H}$ usually infect cats, rodents and seals, respectively [2]. Similar to E. bieneusi, genotyping G. duodenalis also can help us to understand the transmission of this pathogen.

NHPs are also potential reservoir hosts for zoonotic transmission of G. duodenalis [2, 12]. In previous studies, assemblages A, B and $E$ have been identified in humans and NHPs [34]. Among them, assemblage B appears to be most common, while assemblage $\mathrm{E}$ is only occasionally detected [34-38]. Although assemblage A has been further classified into three major sub-assemblages (AIAIII) by sequence analysis of several genetic loci, consistent secondary classification of assemblage B has not been established [2, 12, 39, 40]. Multilocus genotyping (MLG) has been used in several studies to understand the host specificity and zoonotic potential of assemblage $B$ in human and NHPs [41-44]. Controversies exist on the differences in virulence between assemblages A and B in humans [45]. There are no such studies on G. duodenalis in NHPs.

In the present study, we examined the prevalence of $E$. bieneusi and G. duodenalis in fecal specimens from commercial crab-eating macaques (Macaca fascicularis) in Hainan. The genetic diversity of the two pathogen species was assessed using sequence analysis of the ITS ( $E$. bie$n e u s i$ ) and using MLG analysis of the tpi, gdh and $b g$ gene (G. duodenalis). The data generated were used to explore the human-infective potential of these two common gastrointestinal parasites in NHPs.

\section{Methods \\ Specimen collection}

A total of 1452 fecal specimens were collected in April 2016, June 2017, October 2017 and January 2018 from laboratory crab-eating macaques kept on a commercial farm. The farm was founded in 2003 and has been awarded full accreditation from the International Association for Assessment and Accreditation of Laboratory Animal Care (AAALAC) since 2008, with over 20,000 animals at the sampling time. All animals were kept in separate cages $(1 \times 1 \times 1 \mathrm{~m})$ after they were born on the farm, with approximately 30 animals kept in each room of $\sim 60 \mathrm{~m}^{2}$. The cages were elevated $1 \mathrm{~m}$ from the ground so that the feces could fall onto the ground. Every monkey had contact with animals in the neighboring cages. The rooms were cleaned every morning and afternoon to ensure a clean living environment. 
Feed, fruits (apple, banana and peach) and drinking water were regularly distributed by farm staff to each cage every day.

The sampling plan took into consideration the number, age and sex of animals on the farm, and the needed number of positive specimens to generate data for a meaningful assessment of the distribution and human-infective potential of E. bieneusi and G. duodenalis genotypes in these animals. Among the sampled animals, 706 were male, 599 were female and 147 sampled animals had missing information on the sex. The sampled animals belonged to two age groups: 1102 were $1-3$ years-old and 350 were adult monkeys older than 3 years. Regarding the latter, as monkeys over 4 years-old were often sold, the oldest animals sampled in the study were 5 years-old. At the time of sampling, 365 monkeys had loose stools, as defined by runny fecal consistency, and 1087 monkeys were apparently normal. The specimens were stored in $2.5 \%$ potassium dichromate solution at $4{ }^{\circ} \mathrm{C}$ prior to DNA extraction.

\section{DNA extraction}

The stored fecal specimens were washed three times with distilled water by centrifugation at $2000 \times g$ for 10 min. Genomic DNA was extracted from the washed fecal material using the FastDNA SPIN Kit for soil (MP Biomedicals, Santa Ana, CA) [46]. DNA was stored at $-20{ }^{\circ} \mathrm{C}$ before being used in PCR analysis within one year.

\section{Detection and genotyping of Enterocytozoon bieneusi and Giardia duodenalis}

Enterocytozoon bieneusi was detected by nested PCR amplification of a 392-bp fragment of the rRNA gene containing the entire ITS sequence [47]. The genotypes of $E$. bieneusi found in this study were determined by sequencing the PCR products and comparing the sequences obtained from the specimens with the reference sequences from known genotypes. The established genotype nomenclature was used in naming E. bieneusi genotypes identified in this study [15].

Giardia duodenalis was detected by nested PCR amplification of a 530-bp fragment of the tpi gene, a 511-bp fragment of the $b g$ gene and a 599-bp fragment of the $g d h$ gene [48-50]. The specimen was considered G. duodenalis-positive if any of the PCRs generated the expected PCR product. The genotypes of G. duodenalis found in this study were identified by sequencing PCR products from G. duodenalis-positive specimens and comparing sequences obtained with the reference sequences from the known genotypes at each genetic locus.

\section{Sequence analysis}

The secondary PCR products of the ITS, tpi, $b g$ and $g d h$ genes were sequenced in both directions on an ABI 3730 Genetic Analyzer (Applied Biosystems, Foster City, CA, USA). Nucleotide sequences generated were assembled and edited with software ChromasPro v.1.32 (http://technelysium.com.au/ChromasPro.html), and aligned with reference sequences from GenBank by using ClustalX (http://clustal.org).

\section{Phylogenetic analysis}

To explore genetic diversity within the assemblage B of G. duodenalis, tpi, $b g$ and $g d h$ sequences from specimens with complete data at the three genetic loci were concatenated to form multi-locus sequences. They were compared with the reference sequences MLG1MLG15 from NHPs, and Sweh001, Sweh059, Sweh074, Sweh107, Sweh136, Sweh158, ECUST5414, ECUST1710, ECUST4064 and ECUST981 from humans [28, 34, 51]. A maximum likelihood (ML) tree was constructed in MEGA v.6 (https://www.megasoftware.net) using evolutionary distances calculated by the commonly used general time reversible model. The reliability of clusters formed was assessed by bootstrap analysis using 1000 replicates.

\section{Statistical analysis}

Differences in E. bieneusi and G. duodenalis detection rates between groups of different sex, age, or fecal consistency were assessed by using the Chi-square test implemented in SPSS Statistics v.20.0 (IBM Corp., Armonk, NY, USA). The difference was considered significant when $P<0.05$.

\section{Results}

Occurrence of $E$. bieneusi and G. duodenalis in crab-eating macaques

Of the 1452 specimens analyzed, 461 (31.7\%) were positive for $E$. bieneusi. Significantly higher detection rates of $E$. bieneusi were identified in animals with loose stools $(41.4 \%, 151 / 365)$ than animals with normal stools $\left(28.5 \%, 310 / 1087 ; X^{2}=20.83, P<0.0001\right)$, in males (36.5\%, $258 / 706)$ than females $\left(26.7 \%, 160 / 599 ; X^{2}=14.391\right.$, $P=0.0001$ ), and in old animals ( $>3$ years; $38.6 \%, 135 / 350)$ than young animals (1-3 years; 29.6\%, 326/1102; $X^{2}=9.90, P=0.0016$; Table 1).

For G. duodenalis, 362 (24.9\%) specimens were positive by tpi PCR, 315 (21.7\%) by $b g$ PCR and 240 (16.5\%) by gdh PCR. Altogether, 469 (32.3\%) specimens were positive for G. duodenalis in at least one PCR. Significantly higher detection rates of G. duodenalis were found in animals with loose stools $(41.1 \%, 150 / 365)$ than animals with 
Table 1 Distribution of Giardia duodenalis and Enterocytozoon bieneusi genotypes in crab-eating macaques in Hainan, China by fecal consistency, sex and age

\begin{tabular}{|c|c|c|c|c|c|}
\hline \multirow[t]{2}{*}{ Specimen } & \multirow[t]{2}{*}{ Sample size } & \multicolumn{2}{|c|}{ Giardia duodenalis $^{\mathrm{a}}$} & \multicolumn{2}{|c|}{ Enterocytozoon bieneusi } \\
\hline & & No. positive (\%) & Genotype (n) & No. positive (\%) & Genotype ( $n)$ \\
\hline \multirow[t]{8}{*}{ Loose stools ${ }^{b}$} & 365 & $150(41.1)$ & $B(150)$ & $151(41.4)$ & Type IV (74) \\
\hline & & & & & CM1 (40) \\
\hline & & & & & Pongo2 (12) \\
\hline & & & & & Peru8 (11) \\
\hline & & & & & CM2 (8) \\
\hline & & & & & Peru11 (4) \\
\hline & & & & & PigEbITS7 (1) \\
\hline & & & & & $D(1)$ \\
\hline \multirow[t]{8}{*}{ Normal stools } & 1087 & $319(29.3)$ & $B(319)$ & $310(28.5)$ & Type IV (162) \\
\hline & & & & & CM1 (79) \\
\hline & & & & & Peru8 (31) \\
\hline & & & & & Pongo2 (15) \\
\hline & & & & & CM2 (9) \\
\hline & & & & & Peru11 (8) \\
\hline & & & & & CM3 (3) \\
\hline & & & & & $\mathrm{D}(3)$ \\
\hline \multirow[t]{9}{*}{ Male ${ }^{c}$} & 706 & $236(33.4)$ & B (236) & $258(36.5)$ & Type IV (134) \\
\hline & & & & & CM1 (56) \\
\hline & & & & & Peru8 (23) \\
\hline & & & & & Pongo2 (18) \\
\hline & & & & & CM2 (11) \\
\hline & & & & & Peru11 (10) \\
\hline & & & & & $\mathrm{D}(3)$ \\
\hline & & & & & CM3 (2) \\
\hline & & & & & PigEbITS7 (1) \\
\hline \multirow[t]{6}{*}{ Female } & 599 & $181(30.2)$ & $B(181)$ & $160(26.7)$ & Type IV (78) \\
\hline & & & & & CM1 (53) \\
\hline & & & & & Peru8 (14) \\
\hline & & & & & Pongo2 (9) \\
\hline & & & & & CM2 (5) \\
\hline & & & & & $\mathrm{D}(1)$ \\
\hline \multirow[t]{6}{*}{ Unknown } & 147 & $52(35.4)$ & $B(52)$ & $43(29.3)$ & Type IV (24) \\
\hline & & & & & CM1 (10) \\
\hline & & & & & Peru8 (5) \\
\hline & & & & & Peru11 (2) \\
\hline & & & & & CM2 (1) \\
\hline & & & & & CM3 (1) \\
\hline \multirow[t]{9}{*}{$1-3$ years-old ${ }^{d}$} & 1102 & $403(36.6)$ & $B(403)$ & $326(29.6)$ & Type IV (160) \\
\hline & & & & & CM1 (95) \\
\hline & & & & & Peru8 (25) \\
\hline & & & & & Pongo2 (17) \\
\hline & & & & & CM2 (14) \\
\hline & & & & & Peru11 (7) \\
\hline & & & & & $\mathrm{D}(4)$ \\
\hline & & & & & CM3 (3) \\
\hline & & & & & PigEbITS7 (1) \\
\hline
\end{tabular}


Table 1 (continued)

\begin{tabular}{|c|c|c|c|c|c|}
\hline \multirow[t]{2}{*}{ Specimen } & \multirow[t]{2}{*}{ Sample size } & \multicolumn{2}{|c|}{ Giardia duodenalis $^{\mathrm{a}}$} & \multicolumn{2}{|c|}{ Enterocytozoon bieneusi } \\
\hline & & No. positive (\%) & Genotype (n) & No. positive (\%) & Genotype $(n)$ \\
\hline \multirow[t]{6}{*}{$>3$ years-old } & 350 & $66(18.9)$ & $B(66)$ & $135(38.6)$ & Type IV (76) \\
\hline & & & & & CM1 (24) \\
\hline & & & & & Peru8 (17) \\
\hline & & & & & Pongo2 (10) \\
\hline & & & & & Peru11 (5) \\
\hline & & & & & CM2 (3) \\
\hline \multirow[t]{9}{*}{ Total } & 1452 & $469(32.3)$ & B (469) & $461(31.7)$ & Type IV (236) \\
\hline & & & & & CM1 (119) \\
\hline & & & & & Peru8 (42) \\
\hline & & & & & Pongo2 (27) \\
\hline & & & & & CM2 (17) \\
\hline & & & & & Peru11 (12) \\
\hline & & & & & $\mathrm{D}(4)$ \\
\hline & & & & & CM3 (3) \\
\hline & & & & & PigEbITS7 (1) \\
\hline \multicolumn{6}{|c|}{ Giardia duodenalis detection rates were based on PCR analysis of the triosephosphate isomerase (tpi), glutamate dehydrogenase (gdh) and $\beta$-giardin (bg) genes } \\
\hline \multirow{2}{*}{\multicolumn{6}{|c|}{$\begin{array}{l}\text { betection rates of } G \text {. duodenalis }\left(X^{2}=17.25, P<0.0001\right) \text { and } E \text {. bieneusi }\left(X^{2}=20.83, P<0.0001\right) \text { are significantly higher in animals with loose stools than in those with } \\
\text { normal stools } \\
\text { c The detection rate of E. bieneusi is significant higher in males than in females }\left(X^{2}=14.391, P=0.0001\right)\end{array}$}} \\
\hline & & & & & \\
\hline \multicolumn{6}{|c|}{$\begin{array}{l}\mathrm{d} \text { The detection rate of } G \text {. duodenalis is significantly higher in } 1-3 \text { year-old animals than in older animals }\left(X^{2}=38.11, P<0.0001\right) \text {. In contrast, the detection rate of } E \text {. } \\
\text { bieneusi is significantly lower in } 1-3 \text { year-old animals than }>3 \text { years }\left(x^{2}=9.90, P=0.0016\right)\end{array}$} \\
\hline
\end{tabular}

normal stools $\left(29.3 \%, 319 / 1087 ; X^{2}=17.25, P<0.0001\right)$, and in $1-3$ year-old monkeys $(36.6 \%, 403 / 1102)$ than older animals $\left(18.9 \%, 66 / 350 ; X^{2}=38.11, P<0.0001\right)$. Nevertheless, detection rates of $G$. duodenalis were comparable between males $(33.4 \%, 236 / 706)$ and females $(31.2 \%$, 233/746; Table 1).

\section{Distribution of $E$. bieneusi genotypes}

Nine E. bieneusi genotypes were obtained from PCRpositive specimens by sequence analysis, namely Type IV (236/461), CM1 (119/461), Peru8 (42/461), Pongo2 (27/461), CM2 (17/461), Peru11 (12/461), D (4/461), CM3 (3/461) and PigEbITS7 (1/461).

Among them, eight $E$. bieneusi genotypes were found in animals with loose stools, namely Type IV (74/151), CM1 (40/151), Pongo2 (12/151), Peru8 (11/151), CM2 (8/151), Peru11 (4/151), PigEbITS7 (1/151) and D (1/151). Similarly, eight E. bieneusi genotypes were detected in animals with normal stools, namely Type IV (162/310), CM1 (79/310), Peru8 (31/310), Pongo2 (15/310), CM2 (9/310), Peru11 (8/310), CM3 (3/310) and D (3/310). A similar distribution of $E$. bieneusi genotypes was also seen between male and female monkeys as well as young and old monkeys (Table 1).
Distribution of G. duodenalis genotypes and subtypes

Sequence analysis of PCR products from the tpi, $b g$ and $g d h$ genes showed that all 469 G. duodenalis-positive specimens had assemblage B (Table 1). Eight G. duodenalis subtypes were obtained from the 362 PCR-positive specimens at the tpi locus, including four known and four new subtypes. Among them, B-sh01 $(n=108)$, $\mathrm{B} 1(n=75), \mathrm{B} 6(n=27)$ and $\mathrm{B} 2(n=17)$ found in this study were identical to reference sequences JX994245, KC441076, GU564284 and KC441077, respectively. The new subtypes B-hn02 $(n=78), \mathrm{B}-\mathrm{hn04}(n=32)$, B-hn01 $(n=13)$ and B-hn03 $(n=12)$ had one, one, two and one single nucleotide polymorphism (SNP), respectively, compared with the reference sequence MF095053 (Table 2).

Seven G. duodenalis subtypes were present among the 315 PCR-positive specimens at the $b g$ locus, including four known and three new subtypes. Among them, B-CD10 $(n=171), \mathrm{B} 2(n=59), \mathrm{B}-$ Egyh $8(n=58)$ and B-VANC/91/UBC/67 $(n=5)$ found in this study were identical to reference sequences KY696837, MG736242, KC441079 and KM190799, respectively. The new subtypes B-hn08 $(n=20), \mathrm{B}-\mathrm{hn06}(n=1)$ and B-hn07 $(n=1)$ had four, two, and one SNP, respectively, compared with the reference sequence KY696837 (Table 2). 


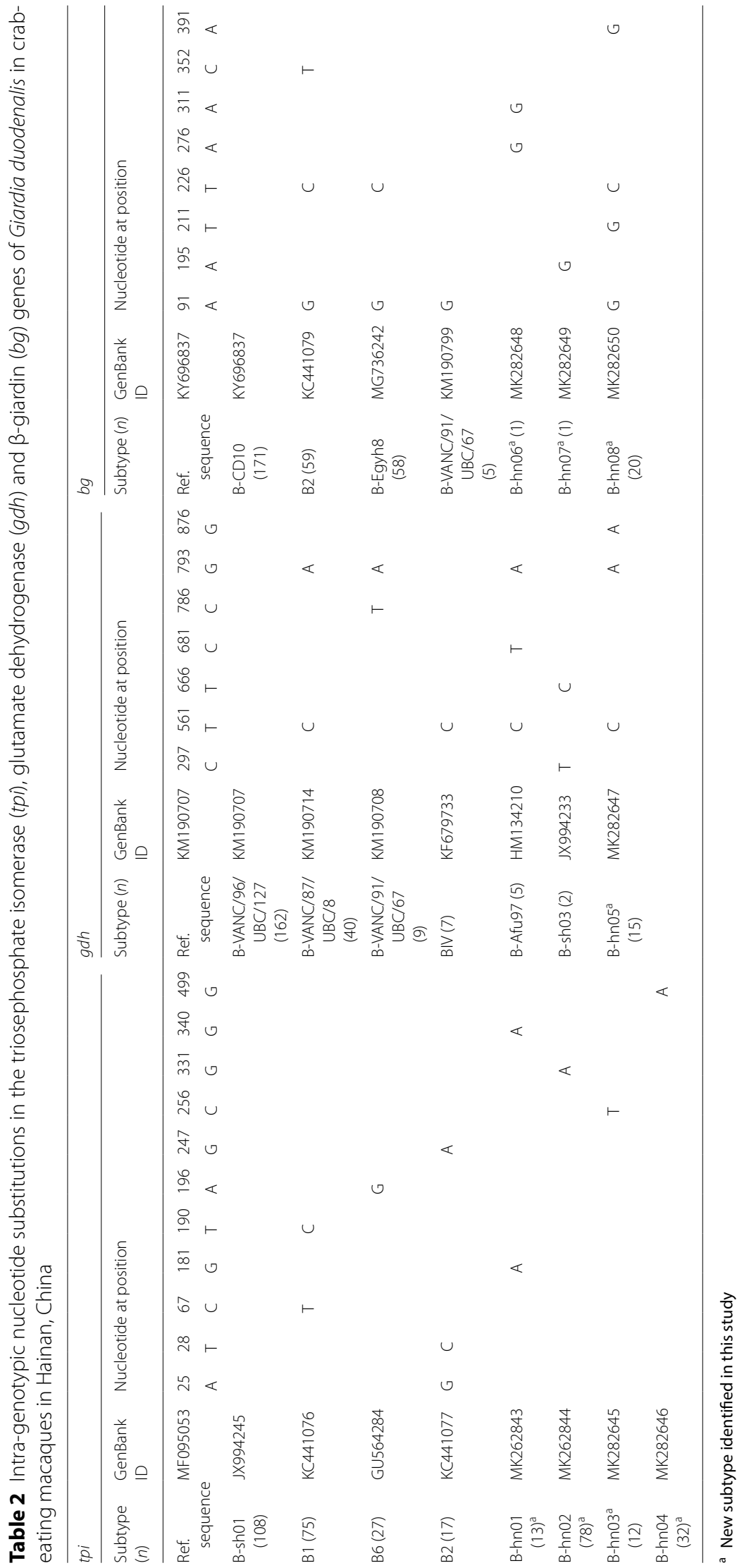


Seven subtypes of G. duodenalis assemblage B were detected among the 240 PCR-positive specimens at the gdh locus, including six known ones and one new subtype. Among them, B-VANC/96/UBC/127 $(n=162), \quad \mathrm{B}-\mathrm{VANC} / 87 / \mathrm{UBC} / 8 \quad(n=40), \quad \mathrm{B}-\mathrm{VANC} / 91 /$ $\mathrm{UBC} / 67(n=9), \mathrm{BIV}(n=7), \mathrm{B}-\mathrm{Afu} 97(n=5)$ and B-sh03 $(n=2)$ found in this study were identical to the reference sequences KM190707, KM190714, KM190708, KF679733, HM134210 and JX994233, respectively. The new subtype B-hn05 $(n=15)$ had three SNPs compared with the reference sequence KM190707 (Table 2).

\section{Multilocus genotyping of assemblage $B$}

Of the 469 specimens positive for $G$. duodenalis assemblage B, 161 were positive by PCR at all three genetic loci. They belonged to 53 MLGs (MLG-B-hn01 to MLG-Bhn53). Among them, MLG-B-hn01 (16.7\%) was the most common, followed by MLG-B-hn02, MLG-B-hn03 and MLG-B-hn04, with frequencies of $7.5 \%, 6.2 \%$, and $5.0 \%$, respectively. In contrast, the frequency of MLG-B-hn05 and MLG-B-hn06 was 4.3\%, the frequency of MLG-Bhn07 and MLG-B-hn08 was 3.7\%, while the remaining MLGs were each seen in fewer than five specimens (Table 3).

\section{Phylogenetic relationship of $G$. duodenalis assemblage B}

Phylogenetic analysis of concatenated sequences of the 53 assemblage B MLGs in this study, and those from previous studies [28, 34, 51] showed that most MLGs from this study were related to MLGs previously found in Old World monkeys (MLG-3, MLG-4, MLG-7, MLG8, MLG-14 and MLG-15). However, one of the MLGs, MLG-B-hn31, seen in one animal, clustered together with MLGs in humans. In addition, MLG-B-hn42 and MLG-B-hn43 were genetically separated from Old World monkeys, ring-tailed lemurs and humans (Fig. 1).

\section{Discussion}

Data from this study suggests that crab-eating macaques in Hainan, China are commonly infected with E. bieneusi. In this study, the detection rate of $E$. bieneusi in these animals was $31.7 \%$ (461/1452). This is higher than the reported detection rates in NHPs in various countries [52-55]. Similarly, it is mostly higher than detection rates in studies of E. bieneusi in NHPs in China [18, 19, 35, 50, 56-59]. Many of the studies reporting low detection rates of $E$. bieneusi in NHPs were performed using wild, captive and zoo animals $[19,35,52,54,55,57]$. The present report represents the first one carried out using a large number of laboratory NHPs.

Crab-eating macaques are apparently infected with zoonotic $E$. bieneusi genotypes. In this study, all nine $E$. bieneusi genotypes detected in these animals belong to
Table 3 Multilocus sequence genotypes of Giardia duodenalis assemblage B in crab-eating macaques in Hainan, China

\begin{tabular}{|c|c|c|c|c|}
\hline \multirow[t]{2}{*}{$M L G s^{a}$} & \multicolumn{3}{|l|}{ Subtype } & \multirow{2}{*}{$\begin{array}{l}\text { No. of } \\
\text { specimens }\end{array}$} \\
\hline & tpi & $g d h$ & $b g$ & \\
\hline MLG-B-hn01 & B-sh01 & B-VANC/96/UBC/127 & $\mathrm{B}-\mathrm{CD} 10$ & 27 \\
\hline MLG-B-hn02 & B-hn02 ${ }^{b}$ & B-VANC/96/UBC/127 & $\mathrm{B}-\mathrm{CD} 10$ & 12 \\
\hline MLG-B-hn03 & B1 & B-VANC/96/UBC/127 & B-Egyh8 & 10 \\
\hline MLG-B-hn04 & B-hn03 ${ }^{b}$ & B-VANC/96/UBC/127 & $\mathrm{B}-\mathrm{CD} 10$ & 8 \\
\hline MLG-B-hn05 & B1 & B-VANC/96/UBC/127 & $\mathrm{B}-\mathrm{CD} 10$ & 7 \\
\hline MLG-B-hn06 & B-sh01 & B-VANC/87/UBC/8 & $\mathrm{B}-\mathrm{CD} 10$ & 7 \\
\hline MLG-B-hn07 & B-hnO2 ${ }^{b}$ & B-VANC/96/UBC/127 & B2 & 6 \\
\hline MLG-B-hn08 & B2 & B-VANC/96/UBC/127 & $\mathrm{B}-\mathrm{CD} 10$ & 6 \\
\hline MLG-B-hn09 & B-sh01 & B-VANC/96/UBC/127 & B2 & 4 \\
\hline MLG-B-hn10 & B-hnO2 ${ }^{b}$ & B-VANC/96/UBC/127 & B-Egyh8 & 4 \\
\hline MLG-B-hn11 & B1 & B-VANC/96/UBC/127 & B2 & 4 \\
\hline MLG-B-hn12 & B-hn01 b & B-VANC/96/UBC/127 & $\mathrm{B}-\mathrm{CD} 10$ & 3 \\
\hline MLG-B-hn13 & B1 & B-VANC/91/UBC/67 & $\mathrm{B}-\mathrm{CD} 10$ & 3 \\
\hline MLG-B-hn14 & B1 & B-hn0 $05^{b}$ & $\mathrm{~B}-\mathrm{CD} 10$ & 3 \\
\hline MLG-B-hn15 & B6 & B-VANC/96/UBC/127 & $\mathrm{B}-\mathrm{CD} 10$ & 3 \\
\hline MLG-B-hn16 & B-sh01 & B-VANC/87/UBC/8 & B-Egyh8 & 3 \\
\hline MLG-B-hn17 & B-sh01 & B-VANC/87/UBC/8 & B2 & 3 \\
\hline MLG-B-hn18 & B1 & B-VANC/87/UBC/8 & $\mathrm{B}-\mathrm{CD} 10$ & 2 \\
\hline MLG-B-hn19 & B6 & B-VANC/91/UBC/67 & B-Egyh8 & 2 \\
\hline MLG-B-hn20 & B-sh01 & B-VANC/96/UBC/127 & B-Egyh8 & 2 \\
\hline MLG-B-hn21 & B-hnO2 ${ }^{b}$ & BIV & B2 & 2 \\
\hline MLG-B-hn22 & B-hnO4 ${ }^{b}$ & B-VANC/96/UBC/127 & B2 & 2 \\
\hline MLG-B-hn23 & B-hnO2 ${ }^{b}$ & B-hn05 b & B-hn08 ${ }^{b}$ & 2 \\
\hline MLG-B-hn24 & B1 & B-VANC/96/UBC/127 & B-hn08 ${ }^{b}$ & 2 \\
\hline MLG-B-hn25 & B-sh01 & B-VANC/91/UBC/67 & $\mathrm{B}-\mathrm{CD} 10$ & 2 \\
\hline MLG-B-hn26 & B-sh01 & B-hn05 b & $\mathrm{B}-\mathrm{CD} 10$ & 2 \\
\hline MLG-B-hn27 & B1 & B-VANC/87/UBC/8 & B2 & 2 \\
\hline MLG-B-hn28 & B1 & B-VANC/87/UBC/8 & B-hn0 $08^{b}$ & 2 \\
\hline MLG-B-hn29 & B-hn01 b & B-VANC/87/UBC/8 & $\mathrm{B}-\mathrm{CD} 10$ & 2 \\
\hline MLG-B-hn30 & B2 & B-hn0 $05^{b}$ & B2 & 1 \\
\hline MLG-B-hn31 & B1 & B-sh03 & $\mathrm{B}-\mathrm{CD} 10$ & 1 \\
\hline MLG-B-hn32 & B1 & B-hn05 & B2 & 1 \\
\hline MLG-B-hn33 & B-sh01 & B-Afu97 & $\mathrm{B}-\mathrm{CD} 10$ & 1 \\
\hline MLG-B-hn34 & B-hnO2 ${ }^{b}$ & BIV & $\mathrm{B}-\mathrm{CD} 10$ & 1 \\
\hline MLG-B-hn35 & B6 & B-hn0 $05^{b}$ & $\mathrm{~B}-\mathrm{CD} 10$ & 1 \\
\hline MLG-B-hn36 & B-hn0 $4^{b}$ & B-VANC/91/UBC/67 & $\mathrm{B}-\mathrm{CD} 10$ & 1 \\
\hline MLG-B-hn37 & B-hnO4 ${ }^{b}$ & B-VANC/91/UBC/67 & B-Egyh8 & 1 \\
\hline MLG-B-hn38 & B1 & B-VANC/87/UBC/8 & B-Egyh8 & 1 \\
\hline MLG-B-hn39 & B6 & B-hn0 $05^{b}$ & B-Egyh8 & 1 \\
\hline MLG-B-hn40 & B1 & B-hn0 $05^{b}$ & B-Egyh8 & 1 \\
\hline MLG-B-hn41 & B2 & BIV & B2 & 1 \\
\hline MLG-B-hn42 & B-sh01 & B-hn0 $05^{b}$ & B2 & 1 \\
\hline MLG-B-hn43 & B-hnO $4^{b}$ & B-hn05 b & B2 & 1 \\
\hline MLG-B-hn44 & B-hnO1 b & B-VANC/96/UBC/127 & B2 & 1 \\
\hline MLG-B-hn45 & B-hn03 ${ }^{b}$ & B-VANC/96/UBC/127 & B2 & 1 \\
\hline MLG-B-hn46 & B-hnO2 ${ }^{b}$ & B-VANC/87/UBC/8 & B-hn08 ${ }^{b}$ & 1 \\
\hline MLG-B-hn47 & B-sh01 & B-VANC/96/UBC/127 & $\begin{array}{c}\text { B-VANC/91/ } \\
\text { UBC/67 }\end{array}$ & 1 \\
\hline
\end{tabular}


Table 3 (continued)

\begin{tabular}{|c|c|c|c|c|}
\hline \multirow[t]{2}{*}{$\mathrm{MLGs}^{\mathrm{a}}$} & \multicolumn{3}{|l|}{ Subtype } & \multirow{2}{*}{$\begin{array}{l}\text { No. of } \\
\text { specimens }\end{array}$} \\
\hline & tpi & $g d h$ & bg & \\
\hline MLG-B-hn48 & B6 & B-Afu97 & $\begin{array}{c}\text { B-VANC/91/ } \\
\text { UBC/67 }\end{array}$ & 1 \\
\hline MLG-B-hn49 & B2 & B-VANC/96/UBC/127 & B-hn08 & 1 \\
\hline MLG-B-hn50 & B-hn0 $04^{b}$ & B-VANC/87/UBC/8 & B-CD10 & 1 \\
\hline MLG-B-hn51 & B6 & B-VANC/87/UBC/8 & B-CD10 & 1 \\
\hline MLG-B-hn52 & B1 & B-VANC/87/UBC/8 & $\begin{array}{c}\text { B-VANC/91/ } \\
\text { UBC/67 }\end{array}$ & 1 \\
\hline MLG-B-hn53 & B1 & BIV & B-Egyh8 & 1 \\
\hline
\end{tabular}

a MLGs are named based on subtypes at the tpi, $g d h$ and $b g$ loci

b New subtype identified in this study the zoonotic Group 1 (Table 1). Among them, Type IV, D, Peru8, Peru11 and PigEbITS7 are known human pathogens in many countries [13, 15, 26-29, 31, 60, 61]. Others such as CM1, CM2 and CM3 have been thus far only found in NHPs in China [18, 19, 62], but this is probably because only a small number of studies have been performed on human $E$. bieneusi infection within the country. The remaining genotype, Pongo2, was reported in China for the first time in this study. This genotype was initially seen in orangutans in Indonesia, indicating that it has the capability to infect a broad range of NHPs [53].



Fig. 1 Phylogenetic relationship of multilocus genotypes (MLGs) of Giardia duodenalis assemblage B inferred by the maximum likelihood analysis of concatenated tpi, gdh and $b g$ nucleotide sequences using genetic distances calculated by the general time reversible model (GTR). Reference sequences (MLG1-15, isolates Sweh001, Sweh059, Sweh074, Sweh107, Sweh136, Sweh158, ECUST1710, ECUST5414, ECUST4064 and ECUST981) used are from the studies by Lebbad et al. [51], Karim et al. [18] and Wang et al. [27]. Bootstrap values greater than 50\% from 1000 replicates are shown on nodes. MLGs identified in the present study are in bold. The scale-bar indicates 50 nucleotide substitutions per 100 nucleotides 
Laboratory crab-eating macaques are also apparently common hosts of G. duodenalis. In this study, the detection rate of G. duodenalis was 32.3\% (469/1452) in Hainan, China. This confirms the prevalence of this pathogen in NHPs in various countries [36, 37, 63, 64] and different areas within China [34, 35, 38, 44, 50, 57, 65]. The very high detection rate of $G$. duodenalis as well as E. bieneusi in the present study could be attributed to the intensive farming of NHPs in this study, which congregates numerous susceptible individuals in confined areas.

To date, assemblages A, B and E of G. duodenalis have been reported in NHPs [34, 38, 50, 54, 65-67]. Among them, assemblage $B$ is the most common genotype in different species of NHPs, including various monkeys, lemurs, gibbons, chimpanzees and gorillas [34, 36-38, $50,57,63-65]$. It is also common in humans in both developing and industrialized countries, and is more common than the other major human-pathogenic genotype, assemblage A [2, 12, 28]. In this study, assemblage $\mathrm{B}$ was the only $G$. duodenalis genotype in the crab-eating macaque. This could have been due to the confined nature of animals in the facility, which limits the introduction of new G. duodenalis genotypes. Nevertheless, a high genetic heterogeneity of assemblage $B$ was seen in animals in the laboratory facility, as revealed by subtype analysis at three genetic loci.

The zoonotic potential of $G$. duodenalis assemblage B in crab-eating macaques was supported by subtype analysis of specimens. Of the eight subtypes detected at the tpi locus, B-sh01 (JX994245) and B6 (GU564284) have been previously found in humans $[28,68]$. Similarly, among the six known subtypes at the $g d h$ locus, B-sh03 (JX994233) and B-VANC/87/UBC/8 (KM190714) have been previously found in humans $[28,69]$. Likewise, among the four known $b g$ subtypes, B-Egyh8 (MG736242) has been previously found in humans [69]. Therefore, many of the known subtypes of G. duodenalis obtained in this study at individual genetic loci had been previously found in humans, supporting the human-pathogenic potential of the assemblage $B$ in crab-eating macaques.

Nevertheless, there appears to be some host segregation within assemblage B of G. duodenalis [34]. In this study, MLG analysis has identified 53 MLGs. Phylogenetic analysis showed that only MLG-B-hn31 is genetically similar to MLGs of assemblage B isolates from humans in China and Sweden $[28,51]$. In contrast, most of other MLGs were genetically related to assemblage B isolates in pig-tailed macaques, rhesus macaques, golden monkeys, yellow baboons and green monkeys, all common Old-World monkeys. They were different from MLGs in ring-tailed lemurs, which are natives of the island nation Madagascar and evolved independently from monkeys and apes.

\section{Conclusions}

In this study, we have shown a frequent occurrence and high genetic diversity E. bieneusi and G. duodenalis subtypes in crab-eating macaques in one commercial laboratory animal facility in Hainan, China. Most of the E. bieneusi genotypes and G. duodenalis assemblage B subtypes are potentially zoonotic. Additional genetic characterizations of these pathogens at other genetic loci, including more conservative ones for $G$. duodenalis, are needed to better understand the transmission of these pathogens and possible occurrence of host segregation within G. duodenalis assemblage B. Measures should be implemented at the commercial facility to reduce the transmission of enteric parasites.

\section{Abbreviations \\ PCR: polymerase chain reaction; MLG: multi-locus genotype; $b g$ : beta-giardia; $g d h$ : glutamate dehydrogenase; tpi: triosephosphate isomerase; ITS: internal transcribed spacer.}

\section{Acknowledgements}

We thank the farm owner and staff for their assistance in sample collection during this study.

\section{Authors' contributions}

YaF and LX conceived and designed the experiments; LC, WJ and YuF performed the experiments; LC, JZ, WJ and YuF analyzed the data; LC, YaF and LX wrote the paper. All authors read and approved the final manuscript.

\section{Funding}

This work was supported by the National Natural Science Foundation of China (31630078, 31602042 and 31425025).

\section{Availability of data and materials}

The data supporting the conclusions of this article are included within the article. Unique sequences generated in this study were submitted to the GenBank database under the accession numbers MK262843-MK262850.

\section{Ethics approval and consent to participate}

The research was reviewed and approved by the Research Ethics Committee of the East China University of Science and Technology, with the approval number of 2015018. Permission was obtained from the farm owner for the specimen collection. Animals were handled in accordance with the Animal Ethics Procedures and Guidelines of the People's Republic of China. The specimens used in the study consisted of fecal droppings collected from the floor of cages in the animal facility, with no animal handling during the specimen collection.

\section{Consent for publication}

Not applicable.

\section{Competing interests}

The authors declare that they have no competing interests.

\section{Author details}

1 State Key Laboratory of Bioreactor Engineering, School of Resource and Environmental, East China University of Science and Technology, Shanghai 200237, China. ${ }^{2}$ Laboratory of Tropical Veterinary Medicine and Vector Biology, Institute of Tropical Agriculture and Forestry, Hainan University, Haikou 570228, Hainan, China. ${ }^{3}$ College of Veterinary Medicine, South China Agricultural University, Guangzhou 510642, China.

Received: 11 March 2019 Accepted: 16 May 2019 Published online: 22 May 2019 


\section{References}

1. Lobo ML, Xiao L, Antunes F, Matos O. Microsporidia as emerging pathogens and the implication for public health: a 10-year study on HIVpositive and -negative patients. Int J Parasitol. 2012;42:197-205.

2. Feng $Y$, Xiao L. Zoonotic potential and molecular epidemiology of Giardia species and giardiasis. Clin Microbiol Rev. 2011;24:110-40

3. Yoder JS, Harral C, Beach MJ. Giardiasis surveillance - United States, 2006-2008. MMWR Surveill Summ. 2010;59:15-25.

4. Khanduja S, Ghoshal U, Agarwal V, Pant P, Ghoshal UC. Identification and genotyping of Enterocytozoon bieneusi among human immunodeficiency virus infected patients. J Infect Public Health. 2017;10:31-40.

5. Tavalla M, Mardani-Kateki M, Abdizadeh R, Nashibi R, Rafie A, Khademva$\tan$ S. Molecular identification of Enterocytozoon bieneusi and Encephalitozoon spp. in immunodeficient patients in Ahvaz, Southwest of Iran. Acta Trop. 2017;172:107-12.

6. Zhang X, Wang Z, Su Y, Liang X, Sun X, Peng S, et al. Identification and genotyping of Enterocytozoon bieneusi in China. J Clin Microbiol. 2011:49:2006-8.

7. Efstratiou A, Ongerth JE, Karanis P. Waterborne transmission of protozoan parasites: review of worldwide outbreaks —an update 2011-2016. Water Res. 2017;114:14-22.

8. Baldursson S, Karanis P. Waterborne transmission of protozoan parasites: review of worldwide outbreaks — an update 2004-2010. Water Res. 2011;45:6603-14

9. Cotte L, Rabodonirina M, Chapuis F, Bailly F, Bissuel F, Raynal C, et al. Waterborne outbreak of intestinal microsporidiosis in persons with and without human immunodeficiency virus infection. J Infect Dis. 1999;180:2003-8

10. Messaoudi I, Estep R, Robinson B, Wong SW. Nonhuman primate model of human immunology. Antioxid Redox Signal. 2011;14:261-73.

11. Mathis A, Weber R, Deplazes P. Zoonotic potential of the microsporidia. Clin Microbiol Rev. 2005;18:423-45.

12. Ryan U, Caccio SM. Zoonotic potential of Giardia. Int J Parasitol. 2013:43:943-56.

13. Matos O, Lobo ML, Xiao L. Epidemiology of Enterocytozoon bieneusi infection in humans. J Parasitol Res. 2012:2012:981424

14. Stentiford GD, Becnel J, Weiss LM, Keeling PJ, Didier ES, Williams BP, et al. Microsporidia-emergent pathogens in the global food chain. Trends Parasitol. 2016;32:336-48.

15. Santin M, Fayer R. Microsporidiosis: Enterocytozoon bieneusi in domesticated and wild animals. Res Vet Sci. 2011;90:363-71

16. Zhang Y, Koehler AV, Wang T, Haydon SR, Gasser RB. New operational taxonomic units of Enterocytozoon in three marsupial species. Parasit Vectors. 2018;11:371.

17. Guo Y, Alderisio KA, Yang W, Cama V, Feng Y, Xiao L. Host specificity and source of Enterocytozoon bieneusi genotypes in a drinking source watershed. Appl Environ Microbiol. 2014;80:218-25.

18. Karim MR, Dong H, Li T, Yu F, Li D, Zhang L, et al. Predomination and new genotypes of Enterocytozoon bieneusi in captive nonhuman primates in zoos in China: high genetic diversity and zoonotic significance. PLOS ONE. 2015;10:e0117991.

19. Karim MR, Wang R, Dong H, Zhang L, Li J, Zhang S, et al. Genetic polymorphism and zoonotic potential of Enterocytozoon bieneusi from nonhuman primates in China. Appl Environ Microbiol. 2014;80:1893-8.

20. Li N, Xiao L, Wang L, Zhao S, Zhao X, Duan L, et al. Molecular surveillance of Cryptosporidium spp., Giardia duodenalis, and Enterocytozoon bieneusi by genotyping and subtyping parasites in wastewater. PLoS Negl Trop Dis. 2012;6:e1809.

21. Thellier M, Breton J. Enterocytozoon bieneusi in human and animals, focus on laboratory identification and molecular epidemiology. Parasite. 2008;15:349-58.

22. Zhang Y, Koehler AV, Wang T, Robertson GJ, Bradbury RS, Gasser RB. Enterocytozoon bieneusi genotypes in people with gastrointestinal disorders in Queensland and Western Australia. Infect Genet Evol. 2018:65:293-9.

23. Li J, Dong H, Wang R, Yu F, Wu Y, Chang Y, et al. An investigation of parasitic infections and review of molecular characterization of the intestinal protozoa in nonhuman primates in China from 2009 to 2015. Int J Parasitol Parasites Wildl. 2017:6:8-15.

24. Akinbo FO, Okaka CE, Omoregie R, Adamu H, Xiao L. Unusual Enterocytozoon bieneusi genotypes and Cryptosporidium hominis subtypes in
HIV-infected patients on highly active antiretroviral therapy. Am J Trop Med Hyg. 2013;89:157-61.

25. Mori H, Mahittikorn A, Thammasonthijarern N, Chaisiri K, Rojekittikhun W, Sukthana Y. Presence of zoonotic Enterocytozoon bieneusi in cats in a temple in central Thailand. Vet Parasitol. 2013;197:696-701.

26. Wang SS, Wang RJ, Fan XC, Liu TL, Zhang LX, Zhao GH. Prevalence and genotypes of Enterocytozoon bieneusi in China. Acta Trop. 2018;183:142-52.

27. Wang L, Zhang H, Zhao X, Zhang L, Zhang G, Guo M, et al. Zoonotic Cryptosporidium species and Enterocytozoon bieneusi genotypes in HIV-positive patients on antiretroviral therapy. J Clin Microbiol. 2013:51:557-63.

28. Wang L, Xiao L, Duan L, Ye J, Guo Y, Guo M, et al. Concurrent infections of Giardia duodenalis, Enterocytozoon bieneusi, and Clostridium difficile in children during a cryptosporidiosis outbreak in a pediatric hospital in China. PLoS Negl Trop Dis. 2013;7:e2437.

29. Wang T, Fan Y, Koehler AV, Ma G, Li T, Hu M, et al. First survey of Cryptosporidium, Giardia and Enterocytozoon in diarrhoeic children from Wuhan, China. Infect Genet Evol. 2017;51:127-31.

30. Yang J, Song M, Wan Q, Li Y, Lu Y, Jiang Y, et al. Enterocytozoon bieneusi genotypes in children in Northeast China and assessment of risk of zoonotic transmission. J Clin Microbiol. 2014;52:4363-7.

31. Santin M, Fayer R. Enterocytozoon bieneusi genotype nomenclature based on the internal transcribed spacer sequence: a consensus. J Eukaryot Microbiol. 2009:56:34-8.

32. Caccio SM, Lalle M, Svard SG. Host specificity in the Giardia duodenalis species complex. Infect Genet Evol. 2018:66:335-45.

33. Heyworth MF. Giardia duodenalis genetic assemblages and hosts. Parasite. 2016:23:13

34. Karim MR, Wang R, Yu F, Li T, Dong H, Li D, et al. Multi-locus analysis of Giardia duodenalis from nonhuman primates kept in zoos in China: geographical segregation and host-adaptation of assemblage $B$ isolates. Infect Genet Evol. 2015:30:82-8.

35. Du SZ, Zhao GH, Shao JF, Fang YQ, Tian GR, Zhang LX, et al. Cryptosporidium spp., Giardia intestinalis, and Enterocytozoon bieneusi in captive non-human primates in Qinling Mountains. Korean J Parasitol. 2015:53:395-402.

36. Johnston AR, Gillespie TR, Rwego IB, McLachlan TL, Kent AD, Goldberg TL. Molecular epidemiology of cross-species Giardia duodenalis transmission in western Uganda. PLoS Negl Trop Dis. 2010:4:e683.

37. Debenham JJ, Tysnes K, Khunger S, Robertson LJ. Occurrence of Giardia, Cryptosporidium, and Entamoeba in wild rhesus macaques (Macaca mulatta) living in urban and semi-rural North-West India. Int J Parasitol Parasites Wildl. 2017;6:29-34.

38. Sricharern W, Inpankaew T, Keawmongkol S, Supanam J, Stich RW, Jittapalapong S. Molecular detection and prevalence of Giardia duodenalis and Cryptosporidium spp. among long-tailed macaques (Macaca fascicularis) in Thailand. Infect Genet Evol. 2016;40:310-4.

39. Geurden T, Levecke B, Caccio SM, Visser A, De Groote G, Casaert S, et al. Multilocus genotyping of Cryptosporidium and Giardia in non-outbreak related cases of diarrhoea in human patients in Belgium. Parasitology. 2009;136:1161-8.

40. Mahdy AK, Surin J, Mohd-Adnan A, Wan KL, Lim YA. Molecular characterization of Giardia duodenalis isolated from Semai Pahang Orang Asli (Peninsular Malaysia aborigines). Parasitology. 2009;136:1237-41.

41. Caccio SM, Beck R, Lalle M, Marinculic A, Pozio E. Multilocus genotyping of Giardia duodenalis reveals striking differences between assemblages A and B. Int J Parasitol. 2008:38:1523-31.

42. Huey CS, Mahdy MA, Al-Mekhlafi HM, Nasr NA, Lim YA, Mahmud R, et al. Multilocus genotyping of Giardia duodenalis in Malaysia. Infect Genet Evol. 2013;17:269-76

43. Wegayehu T, Karim MR, Erko B, Zhang L, Tilahun G. Multilocus genotyping of Giardia duodenalis isolates from calves in Oromia Special Zone, Central Ethiopia. Infect Genet Evol. 2016:43:281-8.

44. Zhong Z, Tian Y, Li W, Huang X, Deng L, Cao S, et al. Multilocus genotyping of Giardia duodenalis in captive non-human primates in Sichuan and Guizhou provinces, Southwestern China. PLoS ONE. 2017;12:e0184913.

45. Xiao L, Feng Y. Molecular epidemiologic tools for waterborne pathogens Cryptosporidium spp. and Giardia duodenalis. Food Waterborne Parasitol. 2017;8-9:14-32. 
46. Jiang J, Alderisio KA, Singh A, Xiao L. Development of procedures for direct extraction of Cryptosporidium DNA from water concentrates and for relief of PCR inhibitors. Appl Environ Microbiol. 2005;71:1135-41.

47. Sulaiman IM, Fayer R, Lal AA, Trout JM, Schaefer FW 3rd, Xiao L. Molecular characterization of microsporidia indicates that wild mammals Harbor host-adapted Enterocytozoon spp. as well as human-pathogenic Enterocytozoon bieneusi. Appl Environ Microbiol. 2003;69:4495-501.

48. Caccio SM, Ryan U. Molecular epidemiology of giardiasis. Mol Biochem Parasitol. 2008:160:75-80.

49. Sulaiman IM, Fayer R, Bern C, Gilman RH, Trout JM, Schantz PM, et al. Triosephosphate isomerase gene characterization and potential zoonotic transmission of Giardia duodenalis. Emerg Infect Dis. 2003;9:1444-52.

50. Ye J, Xiao L, Li J, Huang W, Amer SE, Guo Y, et al. Occurrence of humanpathogenic Enterocytozoon bieneusi, Giardia duodenalis and Cryptosporidium genotypes in laboratory macaques in Guangxi, China. Parasitol Int. 2014:63:132-7.

51. Lebbad M, Petersson I, Karlsson L, Botero-Kleiven S, Andersson JO, Svenungsson B, et al. Multilocus genotyping of human Giardia isolates suggests limited zoonotic transmission and association between assemblage B and flatulence in children. PLoS Negl Trop Dis. 2011;5:e1262

52. Li W, Kiulia NM, Mwenda JM, Nyachieo A, Taylor MB, Zhang X, et al. Cyclospora papionis, Cryptosporidium hominis, and human-pathogenic Enterocytozoon bieneusi in captive baboons in Kenya. J Clin Microbiol. 2011:49:4326-9.

53. Mynarova A, Foitova I, Kvac M, Kvetonova D, Rost M, Morrogh-Bernard $\mathrm{H}$, et al. Prevalence of Cryptosporidium spp., Enterocytozoon bieneusi, Encephalitozoon spp. and Giardia intestinalis in wild, semi-wild and captive orangutans (Pongo abelii and Pongo pygmaeus) on Sumatra and Borneo, Indonesia. PLoS ONE. 2016;11:e0152771.

54. Sak B, Petrzelkova KJ, Kvetonova D, Mynarova A, Shutt KA, Pomajbikova $\mathrm{K}$, et al. Long-term monitoring of microsporidia, Cryptosporidium and Giardia infections in western Lowland Gorillas (Gorilla gorilla gorilla) at different stages of habituation in Dzanga Sangha Protected Areas, Central African Republic. PLoS ONE. 2013;8:e71840.

55. Sak B, Petrzelkova KJ, Kvetonova D, Mynarova A, Pomajbikova K, Modry D, et al. Diversity of microsporidia, Cryptosporidium and Giardia in mountain gorillas (Gorilla beringei beringei) in Volcanoes National Park, Rwanda. PLoS ONE. 2014;9:e109751.

56. Yang H, Lin Y, Li Y, Song M, Lu Y, Li W. Molecular characterization of Enterocytozoon bieneusi isolates in laboratory macaques in north China: zoonotic concerns. Parasitol Res. 2017;116:2877-82.

57. Ye J, Xiao L, Ma J, Guo M, Liu L, Feng Y. Anthroponotic enteric parasites in monkeys in public park, China. Emerg Infect Dis. 2012;18:1640-3.

58. Yu F, Wu Y, Li T, Cao J, Wang J, Hu S, et al. High prevalence of Enterocytozoon bieneusi zoonotic genotype $D$ in captive golden snub-nosed monkey (Rhinopithecus roxellanae) in zoos in China. BMC Vet Res. 2017;13:158.

59. Zhong Z, Li W, Deng L, Song Y, Wu K, Tian Y, et al. Multilocus genotyping of Enterocytozoon bieneusi derived from nonhuman primates in southwest China. PLoS ONE. 2017;12:e0176926.

60. Liu H, Jiang Z, Yuan Z, Yin J, Wang Z, Yu B, et al. Infection by and genotype characteristics of Enterocytozoon bieneusi in HIV/AIDS patients from Guangxi Zhuang autonomous region. China. BMC Infect Dis. 2017;17:684.

61. Lores B, Lopez-Miragaya I, Arias C, Fenoy S, Torres J, del Aguila C. Intestinal microsporidiosis due to Enterocytozoon bieneusi in elderly human immunodeficiency virus-negative patients from Vigo, Spain. Clin Infect Dis. 2002;34:918-21.

62. Garcia RJ, French N, Pita A, Velathanthiri N, Shrestha R, Hayman D. Local and global genetic diversity of protozoan parasites: spatial distribution of Cryptosporidium and Giardia genotypes. PLoS Negl Trop Dis. 2017:11:e0005736.

63. Beck R, Sprong H, Bata I, Lucinger S, Pozio E, Caccio SM. Prevalence and molecular typing of Giardia spp. in captive mammals at the zoo of Zagreb, Croatia. Vet Parasitol. 2011;175:40-6.

64. Berrilli F, Prisco C, Friedrich KG, Di Cerbo P, Di Cave D, De Liberato C. Giardia duodenalis assemblages and Entamoeba species infecting nonhuman primates in an Italian zoological garden: zoonotic potential and management traits. Parasit Vectors. 2011;4:199.

65. Karim MR, Zhang S, Jian F, Li J, Zhou C, Zhang L, et al. Multilocus typing of Cryptosporidium spp. and Giardia duodenalis from non-human primates in China. Int J Parasitol. 2014;44:1039-47.

66. Debenham JJ, Atencia R, Midtgaard F, Robertson LJ. Occurrence of Giardia and Cryptosporidium in captive chimpanzees (Pan troglodytes), mandrills (Mandrillus sphinx) and wild Zanzibar red colobus monkeys (Procolobus kirkii). J Med Primatol. 2015;44:60-5.

67. Lebbad M, Mattsson JG, Christensson B, Ljungstrom B, Backhans A, Andersson JO, et al. From mouse to moose: multilocus genotyping of Giardia isolates from various animal species. Vet Parasitol. 2010;168:231-9.

68. Wang R, Zhang X, Zhu H, Zhang L, Feng Y, Jian F, et al. Genetic characterizations of Cryptosporidium spp. and Giardia duodenalis in humans in Henan, China. Exp Parasitol. 2011;127:42-5.

69. Prystajecky N, Tsui CK, Hsiao WW, Uyaguari-Diaz MI, Ho J, Tang P, et al. Giardia spp. are commonly found in mixed assemblages in surface water, as revealed by molecular and whole-genome characterization. Appl Environ Microbiol. 2015;81:4827-34

\section{Publisher's Note}

Springer Nature remains neutral with regard to jurisdictional claims in published maps and institutional affiliations.
Ready to submit your research? Choose BMC and benefit from:

- fast, convenient online submission

- thorough peer review by experienced researchers in your field

- rapid publication on acceptance

- support for research data, including large and complex data types

- gold Open Access which fosters wider collaboration and increased citations

- maximum visibility for your research: over 100M website views per year

At BMC, research is always in progress.

Learn more biomedcentral.com/submissions 